\title{
CARACTERÍSTICAS CLIINICO-PATOLÓGICAS Y SOBREVIDA EN MUJERES JÓVENES CON CÁNCER CERVICAL: ANÁLISIS RETROSPECTIVO DEL INSTITUTO NACIONAL DE ENFERMEDADES NEOPLÁSICAS
}

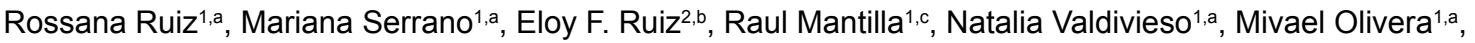 \\ Manuel Alvarez ${ }^{1, d}$, Luis Mas ${ }^{1, a}$, Henry L. Gomez ${ }^{1, a}$
}

\section{RESUMEN}

\begin{abstract}
Objetivos. Determinar las características clínicas, histológicas y los factores pronósticos del cáncer cervical (CC) en pacientes jóvenes peruanas. Materiales y métodos. Análisis retrospectivo de pacientes de 35 años de edad o menos diagnosticadas con CC entre el 2008 y el 2012 en el Instituto Nacional de Enfermedades Neoplásicas. Resultados. 449 pacientes tenían neoplasias epiteliales. Los tipos histológicos principales fueron: carcinoma de células escamosas $(84,9 \%)$, adenocarcinoma $(11,0 \%)$ y carcinoma adenoescamoso $(2,4 \%)$. El tamaño tumoral promedio fue $4,98 \mathrm{~cm}$. También se identificó anemia $(55,7 \%)$, creatinina elevada $(21,2 \%)$ e hidronefrosis $(13,8 \%)$. El $82,3 \%$ de los pacientes presentaron enfermedad localmente avanzada. Los estadios IIB $(47,4 \%)$ y IIIB $(25,8 \%)$ fueron los más comunes. La supervivencia global a 5 años fue de 59,5\% (I, 90,9\%; II, 57,5\%; III, 42,7\% y IV, 13,3\%). La creatinina elevada, la anemia, el tamaño tumoral, el compromiso parametrial y la hidronefrosis fueron factores que afectaron la supervivencia. No se encontró relación significativa entre el tipo histológico y la supervivencia. La presencia de anemia (Hazard Ratio ajustado [HRa]: 2,5; intervalo de confianza al 95\% [IC 95\%]: 1,6-4,0) y la hidronefrosis (HRa: 1,6; IC 95\%: 1,0-4,0) estuvieron independientemente asociados con la supervivencia; asimismo, el compromiso parametrial con (HRa: 3,3; IC 95\%: 1,57,2) o sin (HRa: 2,6; IC 95\%: 1,3-5,3) extensión al hueso pélvico. Conclusiones. El cáncer cervical en jóvenes peruanas es diagnosticado en estadios avanzados. La supervivencia global en cada estadio es similar a la reportada en pacientes mayores. Se confirmó la importancia de los factores convencionales relacionados con el pronóstico. La anemia fue un factor de pronóstico independiente importante que requiere mayores investigaciones.
\end{abstract}

Palabras clave: Neoplasias del cuello uterino; Neoplasias; Perú; Supervivencia; Pronóstico; Adulto joven (fuente: DeCS BIREME).

\section{CLINICAL-PATHOLOGICAL FEATURES AND SURVIVAL IN YOUNG WOMEN WITH CERVICAL CANCER: A RETROSPECTIVE ANALYSIS FROM THE INSTITUTO NACIONAL DE ENFERMEDADES NEOPLÁSICAS}

\begin{abstract}
Objective. To determine the clinical and histological characteristics and prognostic factors of cervical cancer (CC) in young Peruvian patients. Materials and methods. Retrospective analysis of patients younger than 35 years old diagnosed with CC between 2008 and 2012 in the Instituto Nacional de Enfermedades Neoplásicas. Results. 449 patients had epithelial neoplasms. The main histological types were: squamous cell carcinoma (84.9\%), adenocarcinoma (11.0\%) and adenosquamous carcinoma (2.4\%). The average tumor size was $4.98 \mathrm{~cm}$. Anemia $(55.7 \%)$, elevated creatinine $(21.2 \%)$ and hydronephrosis (13.8\%) were also identified. $82.3 \%$ of the patients presented locally advanced disease. Stages IIB (47.4\%) and IIIB (25.8\%) were the most common. Overall 5-year survival was 59.5\% (I, 90.9\%; II, 57.5\%; III, $42.7 \%$ and IV, $13.3 \%$ ). Elevated creatinine, anemia, tumor size, parametrial involvement and hydronephrosis were factors that affected survival. No significant relation was found between histological type and survival. The presence of anemia (adjusted hazard ratio [aHR]: 2.5; 95\% confidence interval [Cl 95\%]: 1.6-4.0) and hydronephrosis (aHR: 1.6; Cl 95\%: 1.0-4.0) were independently associated with survival; likewise, the parametrial commitment with (aHR: 3.3; Cl 95\%: 1.5-7.2) or without (aRH: $2.6 ; \mathrm{Cl}$ 95\%: 1.3-5.3) extension to the pelvic bone. Conclusions. Cervical cancer in young Peruvians is diagnosed in advanced stages. Overall survival in each stage is similar to the reported in older patients. The importance of conventional prognosisrelated factors was confirmed. Anemia was an important independent prognostic factor requiring further investigations.
\end{abstract}

Key words: Uterine cervical neoplasms; neoplasms; Peru; Survival; Prognosis; Young adult (source: MeSH NLM).

\footnotetext{
Instituto Nacional de Enfermedades Neoplásicas. Lima, Perú.

Unidad de Conocimiento y Evidencia CONEVID, Universidad Peruana Cayetano Heredia. Lima, Perú.

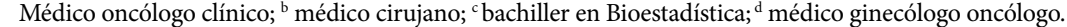

Recibido: 28/01/2017 Aprobado: 24/05/2017 En línea: 28/06/2017
} 


\section{INTRODUCCIÓN}

El cáncer cervical (CC) refleja las diferencias en el acceso a la atención médica alrededor del mundo. A pesar de ser una enfermedad altamente prevenible, continúa siendo un problema de salud pública en las regiones menos desarrolladas, en donde se producen más del $80 \%$ de los casos ${ }^{(1)}$. A nivel global, es la tercera causa de años de vida perdidos ajustados por discapacidad por cáncer en mujeres ${ }^{(1)}$. En Latinoamérica y el Caribe (LAC), el CC ocupa el segundo lugar entre las neoplasias más comúnmente diagnosticadas en mujeres (2). En las mujeres menores de 40 años de la región, no solo es la neoplasia más frecuente, sino también la principal causa de muerte relacionada a cáncer ${ }^{(2)}$. En el Perú, es el cáncer más frecuente en mujeres, con 4636 casos nuevos y 1715 muertes anuales para el $2012^{(2)}$.

A menudo, el CC afecta a mujeres adultas en la cuarta o quinta década de vida, con una edad promedio de 48 años al momento del diagnóstico ${ }^{(3)}$. No obstante, varios estudios epidemiológicos demuestran que la incidencia de CC está aumentando en los grupos etarios más jóvenes ${ }^{(4-7)}$.

Se han descrito que el cáncer cervical en mujeres jóvenes presenta características más agresivas como presentar enfermedad en estadio avanzado al momento del diagnóstico ${ }^{(8)}$, tener un mayor tamaño tumoral ${ }^{(8-10)}$, presentar una tasa más alta de compromiso parametrial ${ }^{(11)}$, nodal ${ }^{(10)}$, y de metástasis a distancia ${ }^{(4)}$. Asimismo, la mayoría de autores concuerda en que en comparación con pacientes mayores, las jóvenes presentan una mayor incidencia de cáncer de células no escamosas ${ }^{(4,11-13)}$. El pronóstico para estos pacientes sigue siendo debatible: si bien la mayor parte de la evidencia respalda resultados inferiores ${ }^{(11,13,14)}$ otros afirman que la edad en sí no afecta la sobrevida ${ }^{(10,15)}$.

Este estudio busca determinar las características clínicopatológicas, supervivencia y factores relacionados al pronóstico de pacientes peruanas jóvenes con $\mathrm{CC}$ que fueron admitidas en el Instituto Nacional de Enfermedades Neoplásicas (INEN), centro de referencia nacional para el manejo de neoplasias, con el fin de evaluar el contexto clínico y epidemiológico de este cáncer en el Perú.

\section{MATERIALES Y MÉTODOS}

\section{DISEÑO DE ESTUDIO}

Estudio retrospectivo. Se hizo un análisis de datos secundarios obtenidos de las historias clínicas del INEN en Lima-Perú.

\section{POBLACIÓN}

La población estuvo conformada por pacientes de 35 años de edad o menos, con diagnóstico de CC, admitidos en el INEN entre enero de 2008 y diciembre de 2012.

\section{MENSAJES CLAVE}

Motivación para realizar el estudio. El cáncer cervical es la neoplasia más frecuente y la principal causa de muerte en las mujeres jóvenes latinoamericanas. Aunque afecta usualmente a aquellas entre los 40 y 50 años, ha aumentado la incidencia en las más jóvenes. Las características clínico-patológicas y de supervivencia en pacientes jóvenes con cáncer cervical en el Perú no se han descrito.

Principales hallazgos. El tipo histológico principal fue el carcinoma de células escamosas, el tipo no escamoso no fue infrecuente. El 82,3\% presentó enfermedad localmente avanzada. La anemia, hidronefrosis y el compromiso parametrial estuvieron asociados a la supervivencia. La sobrevida global en cada estadio fue similar a la reportada para pacientes mayores.

Implicancias. El cáncer cervical en jóvenes peruanas es diagnosticado en estadios avanzados. Requerimos de programas de cribado integrales.

\section{CRITERIOS DE SELECCIÓN}

Se incluyeron todos los casos de pacientes con CC de 35 años de edad o menos admitidas al INEN en dicho periodo. Se excluyeron aquellos casos no incidentes, aquellos cuyas historias clínicas no estuvieron disponibles, pacientes sin confirmación de diagnóstico patológico y pacientes con lesiones preinvasivas del cuello uterino. Se eligió la edad de 35 años como punto de corte debido a que la incidencia de CC se incrementa notablemente por encima de este límite ${ }^{(10)}$.

\section{VARIABLES}

Se incluyó información clínica, epidemiológica y de la historia sexual y reproductiva. Los valores de hemoglobina $\geq 12 \mathrm{~g} / \mu \mathrm{L}$ fueron considerados normales ${ }^{(16)}$. El rango normal para la creatinina fue de 48 a $64 \mathrm{mmol} / \mathrm{L}$ (17). El tamaño tumoral, el compromiso parametrial, vaginal y la extensión a la mucosa de la vejiga y/o recto se determinaron a través del examen físico de acuerdo con el sistema de clasificación de la Federación Internacional de Ginecología y Obstetricia (FIGO) $2009^{(18)}$. La presencia de hidronefrosis se diagnosticó mediante ecografía o tomografía ${ }^{(19)}$. Se definió compromiso nodal cualquier aumento mayor a 1 $\mathrm{cm}$ de los ganglios pélvicos (grupos iliacos y obturadores) o paraaórticos. Los tipos histológicos se determinaron de acuerdo a la clasificación de tumores de los órganos reproductivos femeninos 2014 de la Organización Mundial de la Salud (OMS) ${ }^{(20)}$.

\section{ANÁLISIS ESTADÍSTICO}

El análisis descriptivo se llevó a cabo mediante tablas de distribución de frecuencias. Para el análisis de supervivencia global (SG), el periodo de seguimiento se definió como el tiempo desde el momento del diagnóstico hasta la fecha de muerte o de último contacto. Las curvas de supervivencia se estimaron con el método Kaplan-Meier. Las diferencias entre las curvas 
de acuerdo a variables se establecieron con la prueba log-rank. Los factores de pronóstico se identificaron mediante un modelo regresivo de Cox multivariado. La medida de fuerza de asociación se expresó en hazard ratio (HR), con un intervalo de confianza al 95\% (IC $95 \%)$. Se consideró un nivel de $\mathrm{p}<0,05$ para diferencias significativas. Se utilizó el paquete estadístico SPSS (IBM SPSS Statistics para Windows versión 19.0. Armonk, NY; IBM Corp).

\section{ASPECTOS ÉTICOS}

El protocolo de este estudio fue aprobado por el Comité Institucional de Ética en Investigación del INEN. Se mantuvo la confidencialidad de los datos obtenidos de las historias clínicas.

\section{RESULTADOS}

Entre los años 2008 y 2012, se registraron 8003 casos de CC en el INEN. De estos, $629(7,9 \%)$ tenían 35 años o menos. Se excluyeron 63 casos no incidentes, 25 cuyas historias clínicas no estuvieron disponibles, 68 en los que no se confirmó el diagnóstico patológico y 16 que correspondieron a lesiones preinvasivas del cuello uterino. Finalmente, los 457 casos con diagnóstico patológico de CC fueron seleccionados (Figura 1).

Dentro de los tipos y subtipos histológicos, 449 (98,2\%) pacientes tuvieron neoplasias epiteliales: 388 (84,9\%) correspondieron a carcinomas de células escamosas, $50(11,0 \%)$ a adenocarcinomas y $11(2,4 \%)$ a carcinomas adenoescamosos. De los casos restantes, $8(1,7 \%)$ correspondieron a tumores mesenquimales (sarcoma botrioides y sarcoma alveolar de partes blandas), tumores neuroendocrinos y un caso de tumor de células germinales cervical (Tabla 1).

Los resultados que presentamos a continuación corresponden a los 449 casos de neoplasias epiteliales. Respecto al lugar de procedencia, 267 (59,5\%) pacientes provenían de ciudades de fuera de Lima Metropolitana (provincias y el Callao). El grupo etario más frecuente fue el de 30 a 35 años con $353(78,9 \%)$, seguido del grupo de 25 a 29 años con $72(16,0 \%)$ pacientes y el de 20 a 24 años con $24(5,3 \%)$ pacientes. La media de edad al diagnóstico fue de $32 \pm 3,4$ años. El promedio de edad en la que ocurrió la menarquia, la primera relación sexual y el primer embarazo fue de 13,3 \pm 1,6 años (rango: 9-18 años), 16,7 $\pm 2,5$ años (rango: 8-28 años) y 18,8 años $\pm 3,6$ (rango: $12-34$ años), respectivamente. El promedio del número de parejas sexuales, embarazos y abortos fue de $2 \pm 1,3$ (rango: 1-8), $3 \pm 1,9$ (rango: $0-12$ ) y $1 \pm 1,0$ (rango: $0-11$ ), respectivamente. Seis pacientes $(1,3 \%)$ estaban embarazadas en el momento del diagnóstico y $9(2,0 \%)$ eran VIH positivas.

El tiempo de enfermedad promedio fue de 5,8 $\pm 5,4$ meses (rango: 1-36 meses). La enfermedad fue sintomática en el $96,9 \%$ de los casos, siendo el sangrado genital el síntoma más frecuente con $375(83,5 \%)$ casos, seguido del dolor pélvico con $185(41,2 \%)$ y la descarga vaginal con $125(27,8 \%)$; catorce $(3,1 \%)$ casos fueron asintomáticos. De acuerdo al examen ginecológico, el tamaño tumoral promedio fue de 4,98 $\pm 1,6 \mathrm{~cm}$. La afectación parametrial estuvo presente en el $75,9 \%$ de los casos. Se encontraron anemia, elevación de creatinina e hidronefrosis en el $55,7 \%, 21,2 \%$ y $13,8 \%$

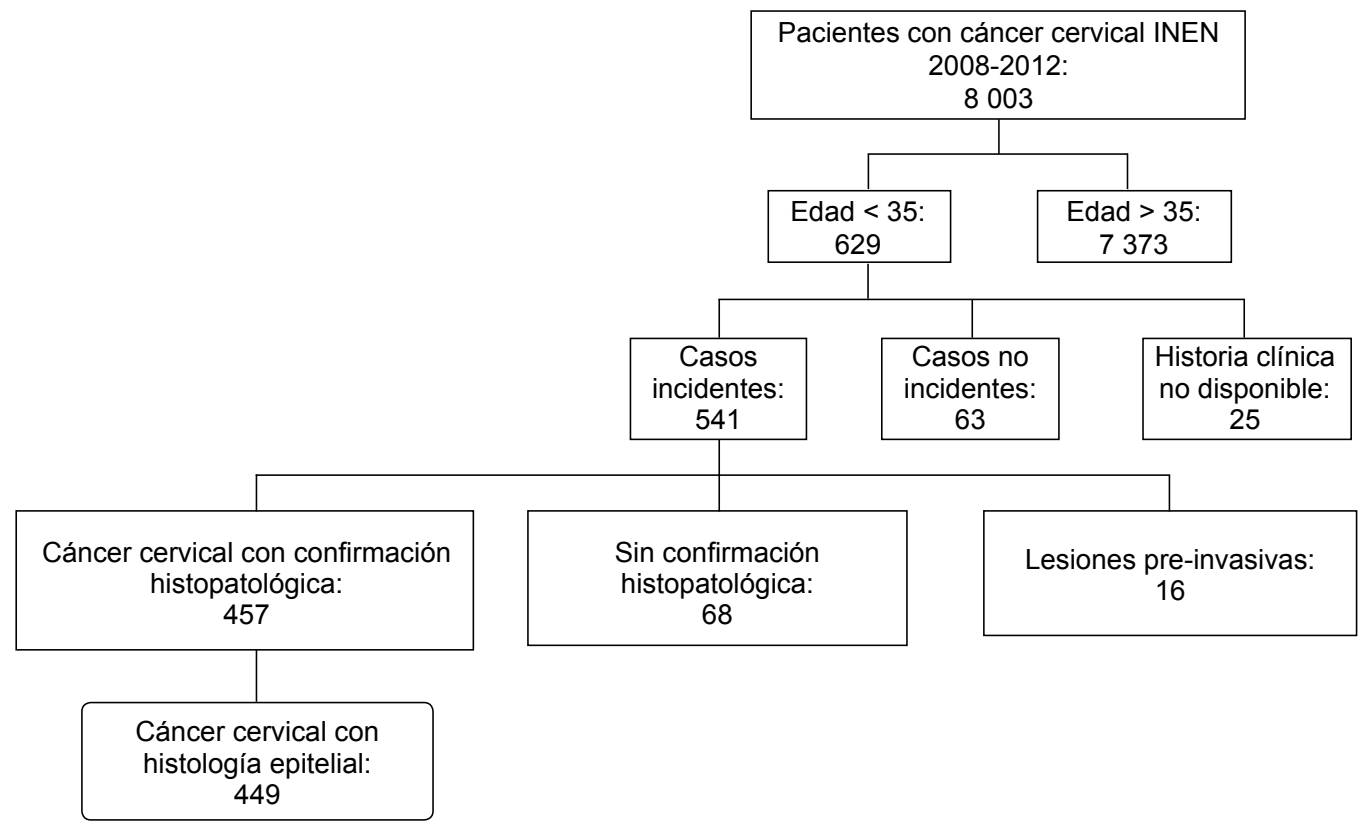

Figura 1. Flujograma de la selección de pacientes 
Tabla 1. Tipos y subtipos histológicos de cáncer cervical $(n=457)$

\begin{tabular}{|rr}
\hline Tipo histológico & Frecuencia (\%) \\
\hline Carcinoma de células escamosas & $388(84,9)$ \\
No queratinizante & $200(51,5)$ \\
Queratinizante & $81(20,9)$ \\
\hline Sarcomatoide & $3(0,8)$ \\
Papilar y escamoso-transicional & $1(0,3)$ \\
\hline No especificado & $103(26,5)$ \\
Adenocarcinoma & $50(11,0)$ \\
\hline Adenocarcinoma mucinoso, & $35(70,0)$ \\
endocervical & $6(12,0)$ \\
Adenocarcinoma endometrioide & $3(6,0)$ \\
Adenocarcinoma de células claras & $1(2,0)$ \\
\hline Tubular o papilar de endocérvix & $1(2,0)$ \\
Desviación mínima & $1(2,0)$ \\
Adenocarcinoma seroso & $3(6,0)$ \\
No especificado & $11(2,4)$ \\
Carcinoma adenoescamoso & $5(1,1)$ \\
Tumores mesenquimales & $2(0,4)$ \\
Neuroendocrino & $1(0,2)$ \\
\hline Misceláneo &
\end{tabular}

de los pacientes, respectivamente. De los 136 pacientes a los que se les realizó tomografía abdominopélvica, $62(13,7 \%)$ tuvieron crecimiento de ganglios linfáticos pélvicos y/o paraaórticos (Tabla 2).

La mayoría de los pacientes $(82,3 \%)$ presentaron enfermedad localmente avanzada (IB2 a IVA). Los estadios más frecuentes fueron IIB y IIIB con $213(47,4 \%)$ y $116(25,8 \%)$ casos, respectivamente. Se encontró una asociación estadísticamente significativa entre el estadio y el lugar de residencia, siendo los estadios III y IV más frecuentes en pacientes provenientes de ciudades diferentes a Lima Metropolitana $(p=0,012)$. Respecto al tratamiento primario por estadio (Tabla 3 ), solo el $14,5 \%$ de los pacientes pudieron ser sometidos a cirugía, mientras que la mayoría recibieron quimioradioterapia concurrente $(42,5 \%)$ o radioterapia $(26,9 \%)$. Cincuenta y seis $(12,5 \%)$ pacientes no recibieron tratamiento alguno.

La SG a 5 años fue de $56,4 \%$ para los 449 pacientes con neoplasias epiteliales. Dentro del grupo de pacientes que sí recibieron algún tipo de tratamiento, el tiempo medio de seguimiento fue de 45 meses y la mediana de supervivencia aún no se ha alcanzado. En este grupo, se estimó una SG a 5 años de 59,5\%.

La SG a 5 años fue de $90,9 \%, 57,5 \%, 42,7 \%$ y $13,3 \%$ para los estadios I, II, III y IV, respectivamente (Figura 2A y Tabla 4). De acuerdo a subestadios, las SG a los 5 años fueron las siguientes: para el estadio IA $(n=20), 100 \%$; IB $(n=68), 88,2 \%$; IIA $(n=5), 100 \%$; IIB $(n=190) 56,3 \%$;
Tabla 2. Características clínico-patológicas

\begin{tabular}{|c|c|}
\hline Característica & Frecuencia (\%) \\
\hline \multicolumn{2}{|l|}{ Estadio FIGO } \\
\hline Estadio I & $94(20,9)$ \\
\hline IA1 & $14(3,1)$ \\
\hline IA2 & $7(1,6)$ \\
\hline IB1 & $43(9,6)$ \\
\hline IB2 & $30(6,7)$ \\
\hline Estadio II & $219(48,7)$ \\
\hline IIA1 & $2(0,4)$ \\
\hline IIA2 & $4(0,9)$ \\
\hline IIB & $213(47,4)$ \\
\hline Estadio III & $118(26,2)$ \\
\hline IIIA & $2(0,4)$ \\
\hline IIIB & $116(25,8)$ \\
\hline Estadio IV & $15(3,3)$ \\
\hline IVA & $3(0,7)$ \\
\hline IVB & $12(2,7)$ \\
\hline Desconocido & $3(0,7)$ \\
\hline \multicolumn{2}{|l|}{ Grado de diferenciación } \\
\hline Bien diferenciado & $24(5,3)$ \\
\hline Moderadamente diferenciado & $321(71,5)$ \\
\hline Poco diferenciado o indiferenciado & $69(15,4)$ \\
\hline No registrado & $35(7,8)$ \\
\hline \multicolumn{2}{|l|}{ Tamaño tumoral } \\
\hline$<4 \mathrm{~cm}$ & $81(18,0)$ \\
\hline$\geq 4 \mathrm{~cm}$ & $315(70,2)$ \\
\hline No registrado & $53(11,8)$ \\
\hline \multicolumn{2}{|l|}{ Compromiso parametrial } \\
\hline Ausente & $105(23,4)$ \\
\hline $\begin{array}{l}\text { Compromiso sin extensión al hueso } \\
\text { pélvico }\end{array}$ & $223(49,7)$ \\
\hline $\begin{array}{l}\text { Compromiso con extensión al hueso } \\
\text { pélvico }\end{array}$ & $118(26,3)$ \\
\hline No registrado & $3(0,7)$ \\
\hline \multicolumn{2}{|l|}{ Compromiso vaginal } \\
\hline Ausente & $205(45,7)$ \\
\hline Tercio superior o medio & $218(48,6)$ \\
\hline Tercio inferior & $21(4,7)$ \\
\hline No registrado & $5(1,1)$ \\
\hline \multicolumn{2}{|l|}{ Compromiso de la mucosa de la vejiga y/o recto } \\
\hline Ausente & $433(96,4)$ \\
\hline Presente & $13(2,9)$ \\
\hline No registrado & $3(0,7)$ \\
\hline \multicolumn{2}{|l|}{ Compromiso ganglionar } \\
\hline Sin agrandamiento ganglionar & $74(16,48)$ \\
\hline $\begin{array}{l}\text { Ganglios pélvicos (grupos iliacos y } \\
\text { obturadores) }\end{array}$ & $44(9,7)$ \\
\hline $\begin{array}{l}\text { Ganglios paraaórticos o pélvicos + } \\
\text { paraaórticos }\end{array}$ & $18(4)$ \\
\hline Desconocido & $313(69,7)$ \\
\hline \multicolumn{2}{|l|}{ Hemoglobina } \\
\hline$\geq 12 \mathrm{~g} / \mathrm{dL}$ & $165(36,7)$ \\
\hline$<12 \mathrm{~d} / \mathrm{dL}$ & $250(55,7)$ \\
\hline No registrado & $34(7,6)$ \\
\hline \multicolumn{2}{|l|}{ Creatinina } \\
\hline$<65 \mu \mathrm{mol} / \mathrm{L}$ & $321(71,5)$ \\
\hline$\geq 65 \mu \mathrm{mol} / \mathrm{L}$ & $95(21,2)$ \\
\hline No registrado & $33(7,3)$ \\
\hline \multicolumn{2}{|l|}{ Hidronefrosis } \\
\hline Ausente & $372(82,9)$ \\
\hline Presente & $62(13,8)$ \\
\hline No registrado & $15(3,3)$ \\
\hline
\end{tabular}


Tabla 3. Tratamiento primario por estadio de los carcinomas epiteliales

\begin{tabular}{|c|c|c|c|c|c|c|}
\hline \multirow{2}{*}{$\begin{array}{l}\text { Subestadio } \\
\text { tumoral }\end{array}$} & Cirugía & $\begin{array}{l}\text { Quimioterapia } \\
\text { neoadyuvante }\end{array}$ & Radioterapia & $\begin{array}{l}\text { Quimioradiación } \\
\text { concurrente }\end{array}$ & Quimioterapia & Ninguna \\
\hline & n (\%) & n (\%) & n (\%) & n (\%) & n (\%) & $n(\%)$ \\
\hline IA1 & $13(92,9)$ & 0 & 0 & 0 & 0 & $1(7,1)$ \\
\hline IA2 & $7(100,0)$ & 0 & 0 & 0 & 0 & 0 \\
\hline IB1 & $39(90,7)$ & 0 & 0 & 0 & 0 & $4(9,3)$ \\
\hline IB2 & $5(16,1)$ & $13(41,9)$ & $2(6,5)$ & $9(29,0)$ & 0 & $2(6,5)$ \\
\hline IIA1 & $1(50,0)$ & 0 & 0 & 0 & 0 & $1(50,0)$ \\
\hline IIA2 & 0 & $1(25,0)$ & 0 & $3(75,0)$ & 0 & 0 \\
\hline IIB & 0 & 0 & $59(27,6)$ & $132(61,7)$ & 0 & $23(10,7)$ \\
\hline IIIA & 0 & 0 & $1(50,0)$ & $1(50,0)$ & 0 & 0 \\
\hline IIIB & 0 & 0 & $48(41,4)$ & $42(36,2)$ & $1(0,9)$ & $25(21,6)$ \\
\hline IVA & 0 & 0 & $2(66,7)$ & $1(33,3)$ & 0 & 0 \\
\hline IVB & 0 & 0 & $9(69,2)$ & $3(23,1)$ & $1(7,7)$ & 0 \\
\hline Total & $65(14,5)$ & $14(3,1)$ & $121(26,9)$ & $191(42,5)$ & $2(0,4)$ & $56(12,5)$ \\
\hline
\end{tabular}

IIIB ( $n=91), 43,7 \%$ y IVA $(n=3), 33,3 \%$. Para el estadio IIIA $(n=2)$ la SG fue del $0 \%$ a los 11 meses y para el estadio IVB $(n=12)$ de $8,3 \%$ a los 36 meses (Figura 2B y Tabla 4). Se encontró una diferencia significativa en la supervivencia de acuerdo al estadio $(p<0,001)$.

En el análisis univariado, la elevación de la creatinina, la presencia de anemia, el tamaño tumoral $\geq 4 \mathrm{~cm}$, la afectación parametrial, la hidronefrosis y la presencia de ganglios mayores de $1 \mathrm{~cm}$ en la tomografía abdominopélvica fueron factores que afectaron la supervivencia. No se encontró asociación significativa entre la supervivencia y el tipo histológico o grado de diferenciación. En el análisis multivariado, la anemia (HR: 2,5; IC 95\%: 1,6-4,0), la afectación parametrial

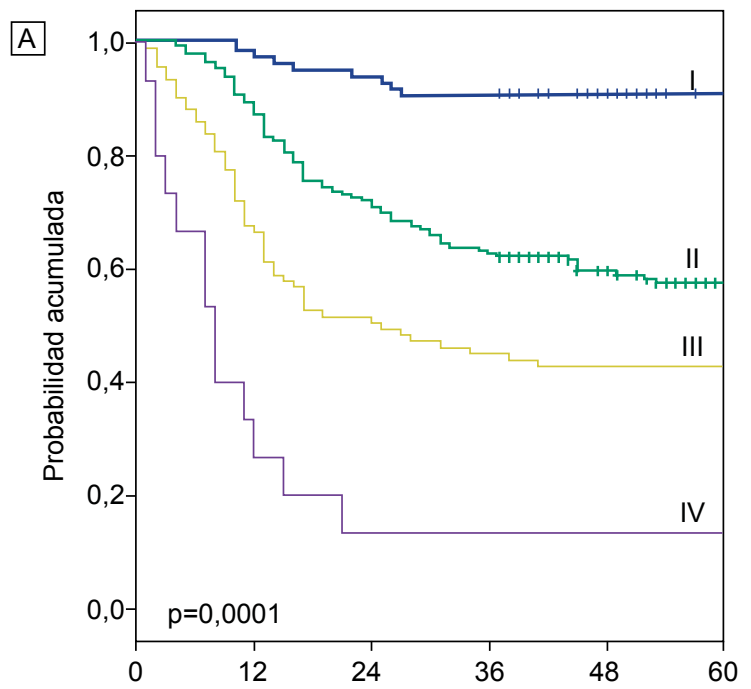

Tiempo desde el diagnóstico (meses)
(HR: 2,6; IC 95\%: 1,3-5,3) y la hidronefrosis (HR: 1,6; IC 95\%: 1,0-2,6) se asociaron de forma significativa e independiente con la SG (Tabla 5).

Doscientos dos pacientes fallecieron. De estos, $77,23 \%$ se perdieron de vista con evidencia de progresión de la enfermedad y fallecieron poco después; sin embargo, no se pudo determinar la causa directa de la muerte. De los pacientes con causas directas de muerte registradas, todos, excepto uno $(0,50 \%)$, murieron debido al CC. Las causas más frecuentes fueron caquexia $(9,41 \%)$, anemia aguda $(4,95 \%)$ y enfermedad renal crónica reagudizada $(3,96 \%)$. Otras causas $(3,95 \%)$ incluyeron hemorragia, pielonefritis y fenómenos trombóticos.

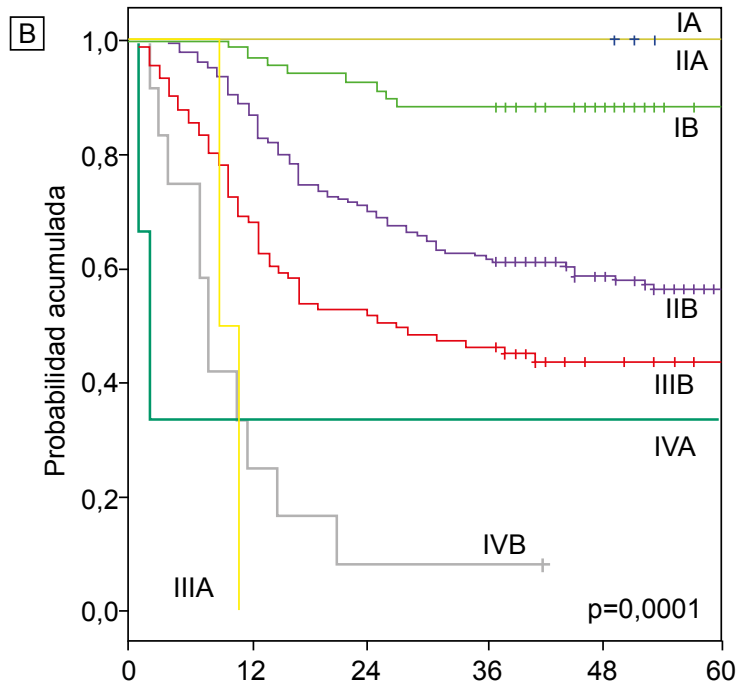

Tiempo desde el diagnóstico (meses)

Figura 2. Curvas de Kaplan-Meier. A. Sobrevida global de acuerdo a estadios FIGO de los pacientes con tumores epiteliales tratados. B. Sobrevida global según subestadios FIGO en pacientes con tumores epiteliales tratados. 
Tabla 4. Estimaciones de la sobrevida global de acuerdo a estadios FIGO de los pacientes con tumores epiteliales tratados

\begin{tabular}{|c|c|c|c|c|}
\hline \multirow{2}{*}{$\begin{array}{l}\text { Estadio } \\
\text { tumoral }\end{array}$} & \multicolumn{3}{|c|}{ Sobrevida global (\%) } & \multirow{2}{*}{ Valor $p$} \\
\hline & 1 año & 3 años & 5 años & \\
\hline $\mathrm{I}$ & 97,7 & 90,9 & 90,9 & \multirow{12}{*}{$<0,001$} \\
\hline IA & 100,0 & 100,0 & 100,0 & \\
\hline IB & 97,1 & 88,2 & 88,2 & \\
\hline II & 87,2 & 62,6 & 57,5 & \\
\hline$\| A$ & 100,0 & 100,0 & 100,0 & \\
\hline IIB & 86,8 & 61,6 & 56,3 & \\
\hline III & 66,7 & 45,2 & 42,7 & \\
\hline IIIA & $0,0^{*}$ & & & \\
\hline IIIB & 68,1 & 46,2 & 43,7 & \\
\hline IV & 26,7 & 13,3 & 13,3 & \\
\hline IVA & 33,3 & 33,3 & 33,3 & \\
\hline IVB & 25,0 & 8,3 & & \\
\hline
\end{tabular}

* Estimado a los 11 meses

\section{DISCUSIÓN}

Este análisis representa el primer intento de estudio del CC en pacientes jóvenes peruanas. Se analizaron retrospectivamente los datos de 5 años del INEN, el centro de cáncer de referencia más importante del Perú.

Los pacientes jóvenes representaron el 7,8\% de todos los casos de CC diagnosticados durante el período de estudio, porcentaje que se encuentra dentro del rango de $7^{(21)}$ a $25 \%{ }^{(22)}$ reportado en la literatura. Diversos estudios afirman que la incidencia de CC está aumentando entre las mujeres jóvenes. Un estudio desarrollado en Inglaterra, que evaluó registros de CC entre 1992 y 2006, informó que la incidencia de CC entre las mujeres de 20 a 29 años aumentó en $2,2 \%$, anualmente ${ }^{(6)}$. Otro estudio realizado en China encontró que la prevalencia de $\mathrm{CC}$ en mujeres de 35 años, o menos, aumentó constantemente de 2,8 a $15,7 \%$ en el período entre 1975 y $2009{ }^{(4)}$. Este fenómeno estaría relacionado con la iniciación sexual cada vez más temprana ${ }^{(23)} \mathrm{o}$ a la disminución del número de casos con CC en pacientes de edad avanzada y el consecuente incremento relativo en pacientes jóvenes (21). Por otro lado, un estudio danés que evaluó las tendencias en CC de 1997 al 2012 concluyó que, si bien su programa de tamizaje reducía la incidencia global de CC, no disminuía significativamente la incidencia en mujeres jóvenes ${ }^{(7)}$.

Nuestra población joven se caracterizó por una edad precoz de iniciación de la actividad sexual y reproductiva. El mecanismo por el cual el inicio de la actividad sexual y el primer embarazo a edades tempranas podrían influir en el riesgo de carcinogénesis cervical en la unión escamo-columnar, está relacionado con el mayor riesgo de infección por el virus del papiloma humano (VPH), responsable del desarrollo de virtualmente todos los casos de CC. Esto es debido a la influencia de las hormonas esteroideas en dicha infección y a la respuesta inmune inmadura del huésped ${ }^{(24)}$.

Estudios previos sugieren que la distribución histológica del CC varía entre pacientes jóvenes y mayores, siendo mayor la probabilidad de encontrar carcinomas no escamosos entre las jóvenes, con una frecuencia del $13,1 \%{ }^{(13)}$ al 19,3\% ${ }^{(5)}$. De hecho, un estudio que incluyó a más de 2500 pacientes encontró que el carcinoma no escamoso representaba el 14,9 y el $6,8 \%$ de los casos, en pacientes menores y mayores de 35 años de edad, respectivamente ${ }^{25)}$. En nuestra serie, el carcinoma no escamoso representó el $13,3 \%$ de los casos. A pesar de que muchos estudios indican una tendencia hacia peores resultados para el carcinoma no escamoso en comparación con el carcinoma de células escamosas ${ }^{(5,8,26,27)}$, otros ${ }^{(28-30)}$, así como nuestra serie, no encontraron diferencias en la supervivencia por tipo histológico.

La enfermedad localmente avanzada (IB2 a IVA), habitualmente no susceptible de cirugía, representó la gran mayoría de nuestros casos $(82,3 \%)$, siendo los estadios II $(48,7 \%)$ y III $(26,2 \%)$, los más frecuentes. Por otra parte, se encontró que la presentación en estadios III y IV fue significativamente más común en los pacientes de provincia. Un estudio peruano previo que evaluó a 2547 pacientes, encontró que las mujeres fueron diagnosticadas en estadio II $(44,11 \%)$ y III $(39,3 \%)$, principalmente ${ }^{(31)}$. Otros estudios retrospectivos en LAC, no restringidos a la población joven ${ }^{(30,32)}$, describieron también esta realidad. Una situación radicalmente diferente fue mostrada por una serie japonesa de 441 pacientes menores de 35 años en la que el $84,8 \%$ de los casos fueron diagnosticados en estadio I ${ }^{(5)}$.

Una proporción tan alta de diagnósticos en etapas avanzadas da muestra clara del poco éxito de nuestra estrategia nacional de cribado. Esta situación es la misma para la mayoría de los países de LAC, donde los programas de tamizaje basados en citología tienen menos probabilidades de tener éxito debido a los sistemas de atención de salud fragmentados que carecen de recursos e infraestructura adecuados. Además de la cobertura subóptima de la población objetivo $-58,2 \%$ en el Perú (33)-, limitaciones relacionadas con el adecuado diagnóstico citológico, el seguimiento y el acceso al tratamiento, así como la falta de concientización y las barreras socioculturales, deben ser abordadas sistemáticamente dentro de un programa de cribado integral ${ }^{(34)}$. La prevención primaria con inmunización contra el VPH para las niñas es altamente efectiva y se ha introducido en la mayoría de los países latinoamericanos, así como desde 2011 en Perú ${ }^{(34)}$, sin embargo, no se espera que esta estrategia reduzca sustancialmente la incidencia de CC sino hasta dentro de algunas décadas, resaltando aun más la necesidad de implementar un programa de tamizaje integral. 
Tabla 5. Análisis univariado y multivariado de factores pronóstico para sobrevida global en pacientes con tumores epiteliales tratados

\begin{tabular}{|c|c|c|c|c|}
\hline Característica & $\begin{array}{l}\text { HR crudo } \\
\text { (IC 95\%) }\end{array}$ & Valor $p$ & $\begin{array}{l}\text { HR ajustado } \\
\text { (IC } 95 \%)\end{array}$ & Valor $p$ \\
\hline \multicolumn{5}{|l|}{ Creatinina } \\
\hline$<65 \mu \mathrm{mol} / \mathrm{L}$ & 1 & & 1 & \\
\hline$\geq 65 \mu \mathrm{mol} / \mathrm{L}$ & $1,5(1,1-2,2)$ & 0,014 & $1,1(0,7-1,7)$ & 0,676 \\
\hline \multicolumn{5}{|l|}{ Hemoglobina } \\
\hline$\geq 12 \mathrm{~g} / \mathrm{dL}$ & 1 & & 1 & \\
\hline$<12 \mathrm{~g} / \mathrm{dL}$ & $4,0(2,7-6,0)$ & $<0,001$ & $2,5(1,6-4,0)$ & $<0,001$ \\
\hline \multicolumn{5}{|l|}{ Tamaño tumoral } \\
\hline$<4 \mathrm{~cm}$ & 1 & & 1 & \\
\hline$\geq 4 \mathrm{~cm}$ & $3,4(1,9-6,0)$ & $<0,001$ & $1,3(0,7-2,5)$ & 0,463 \\
\hline \multicolumn{5}{|l|}{ Compromiso parametrial } \\
\hline Ausente & 1 & & 1 & \\
\hline Compromiso sin extensión al hueso pélvico & $4,3(2,4-7,7)$ & $<0,001$ & $2,6(1,3-5,3)$ & 0,007 \\
\hline Compromiso con extensión al hueso pélvico & $6,9(3,8-12,7)$ & $<0,001$ & $3,3(1,5-7,2)$ & 0,002 \\
\hline \multicolumn{5}{|l|}{ Hidronefrosis } \\
\hline Ausente & 1 & & 1 & \\
\hline Presente & $2,8(1,9-4,0)$ & $<0,001$ & $1,6(1,0-2,6)$ & 0,041 \\
\hline \multicolumn{5}{|l|}{ Compromiso ganglionar* } \\
\hline Sin agrandamiento ganglionar & 1 & & & \\
\hline Ganglios pélvicos (grupos iliacos y obturadores) & $2,5(1,6-4,3)$ & 0,003 & -- & -- \\
\hline Ganglios paraaórticos o pélvicos + paraaórticos & $2,8(1,4-5,7)$ & 0,003 & -- & -- \\
\hline \multicolumn{5}{|l|}{ Tipo histológico* } \\
\hline Carcinoma escamoso & 1 & & & \\
\hline Adenocarcinoma & $0,7(0,4-1,2)$ & 0,199 & -- & -- \\
\hline Carcinoma adenoescamoso & $1,9(0,8-4,3)$ & 0,120 & -- & -- \\
\hline \multicolumn{5}{|l|}{ Grado de diferenciación * } \\
\hline Bien diferenciado & 1 & & & \\
\hline Moderadamente diferenciado & $0,6(0,3-1,1)$ & 0,075 & -- & -- \\
\hline Poco diferenciado o indiferenciado & $0,2(0,3-1,2)$ & 0,150 & -- & -- \\
\hline
\end{tabular}

* No explorado por cantidad insuficiente de pacientes.

Modelo de regresión de Cox. HR: hazard ratio

La información sobre el pronóstico de CC en este grupo de edad es controversial. Numerosos investigadores han tratado de mostrar el efecto de la edad sobre la supervivencia. La serie con el mayor número de pacientes jóvenes fue publicada en 1988 por Meanwell (26). Se incluyeron 10022 pacientes tratados entre 1957 y 1981 de los cuales 1605 fueron menores de 40 años. Se encontró una diferencia estadísticamente significativa en la supervivencia estadio por estadio, favoreciendo a los pacientes más jóvenes ${ }^{(26)}$. Kosary et al. demostraron que la SG y por estadios a 5 años fue mayor en las mujeres menores de 30 años y esta declinó constantemente a medida que aumentaba la edad ${ }^{(35)}$. Otros estudios comparativos más modernos no han encontrado diferencias en la supervivencia entre pacientes jóvenes y mayores ${ }^{(12,36,37)}$. Por el contrario, una publicación reciente que comparó a 831 pacientes menores de 35 años y 1737 mayores de 35 años, informó que la tasa de supervivencia a los 5 años fue del 75 y $39,8 \%$ en los pacientes jóvenes y del 84 y $54 \%$ en el grupo control ${ }^{25)}$. Del mismo modo, en un estudio pareado por estadio y tratamiento, encontró que las mujeres menores de 35 años tenían un peor pronóstico en comparación 
con las pacientes mayores de 35 años ${ }^{(9)}$. En nuestro análisis, en los estadios IB, IIB y IIIB, estadios con un número considerable de sujetos $\mathrm{y}$, por lo tanto, con mayor posibilidad de que los resultados sean representativos, las tasas de supervivencia a 5 años fueron similares a las descritas en los reportes del American Joint Committee on Cancer (AJCC) ${ }^{(38)}$ y la FIGO ${ }^{(39)}$. Por lo tanto, el presente estudio retrospectivo sugiere que el pronóstico en los pacientes más jóvenes no es inferior. Claramente, un grupo comparativo de pacientes mayores dentro de nuestra misma institución es necesario para verificar esta hipótesis.

Los estudios que evalúan los factores pronósticos específicamente en la población joven son muy escasos. En el análisis univariado, nuestra serie confirmó la relevancia de los factores pronósticos convencionales para la población joven: tamaño tumoral, compromiso parametrial, niveles de creatinina e hidronefrosis. Además, el agrandamiento patológico de los ganglios linfáticos pélvicos y/o paraaórticos, evaluados por tomografía, también tuvo un efecto perjudicial sobre la supervivencia. Se ha demostrado que la presencia de ganglios linfáticos mayores de $1 \mathrm{~cm}$ medido por tomografía es un factor pronóstico independiente para SG y control local en pacientes con CC tratados con radioterapia (40) o quimioradiación concurrente (41). A partir del estadio IB2 en adelante, a menos que se lleve a cabo una estadificación quirúrgica, se recomienda la evaluación radiológica de los ganglios linfáticos, ya que podría alterar la planificación del tratamiento.

Los factores independientemente asociados con la supervivencia fueron la afectación parametrial, la presencia de hidronefrosis y de anemia. Asimismo, la hidronefrosis no fue una presentación infrecuente en nuestros pacientes. Una evaluación retrospectiva de 539 pacientes en estadio IIIB tratados en cuatro ensayos prospectivos realizados por el Gynecologic Oncology Group (GOG) encontró que la mediana de supervivencia en pacientes sin hidronefrosis fue significativamente mayor (69,5 meses) que aquellos que sí la presentaban (31,5 meses), independientemente de recibir dosis similares de radiación y quimioterapia basada en cisplatino ${ }^{(42)}$. En nuestra serie, los pacientes con hidronefrosis tuvieron una SG a 5 años de $33 \%$ versus $64 \%$ para los pacientes sin esta característica. Dado que está asociada a una morbilidad importante y una supervivencia menor, la evaluación de la hidronefrosis, que es objetiva y reproducible, ayuda a la estratificación del riesgo y a definir estrategias especiales de tratamiento. Es necesario recalcar que tanto la hidronefrosis como la afectación parametrial se integran en el sistema de estadificación FIGO.

Por otro lado, la anemia, un factor poco considerado, también mostró significancia pronóstica en nuestra serie.
Las tasas de SG a 5 años fue de $82 \%$ versus $44 \%$ para pacientes con valores de hemoglobina $\geq 12 \mathrm{~g} / \mathrm{dL}$ y $<12 \mathrm{~g} / \mathrm{dL}$, respectivamente. En el análisis multivariado, la presencia de anemia siguió siendo un factor negativo para la SG. Los pacientes con anemia tuvieron dos veces más probabilidades de morir (HR: 2,5; IC 95\%, 1,6-3,9). Esto podría explicarse por el hecho de que la hipoxia intratumoral, secundaria a la anemia, perjudicaría la sensibilidad tumoral a la radio y quimioterapia al privar a las células tumorales del oxígeno molecular esencial para lograr la máxima actividad citotóxica ${ }^{(43)}$. Otros estudios han identificado a la anemia como un factor pronóstico independiente para la supervivencia libre de enfermedad y SG en pacientes con CC ${ }^{(37,44)}$. Un ensayo aleatorizado que evaluó el soporte transfusional en pacientes con $\mathrm{CC}$ tratados con radioterapia en el Hospital Princess Margaret, demostró que los pacientes anémicos que fueron transfundidos a un nivel de hemoglobina de al menos $12,5 \mathrm{~g} / \mathrm{dL}$ tenían una menor tasa de recurrencia locorregional que aquellos que se mantuvieron con hemoglobina de al menos $10 \mathrm{~g} / \mathrm{dL}^{(45)}$. El hecho de que la presencia de anemia esté asociada independientemente con una mayor mortalidad por CC requiere ser explorado, pues es un parámetro fácilmente cuantificable que podría identificar un subgrupo de pacientes con mal pronóstico para quienes la corrección oportuna de la anemia y las estrategias de mantenimiento podrían ser beneficiosas.

Nuestro estudio posee algunas limitaciones. En primer lugar, la información fue obtenida retrospectivamente a partir de la revisión de historias clínicas y, en algunos casos, hubo datos faltantes. Así mismo, el tiempo de seguimiento, aunque largo, fue insuficiente ya que la mediana de SG aún no se ha alcanzado. No obstante, la fuente utilizada es representativa y permite caracterizar el panorama nacional. Nuestra dirección futura es realizar un análisis prospectivo y comparativo con un grupo de pacientes mayores de nuestra institución y profundizar en el valor de hemoglobina como factor pronóstico independiente y modificable.

En conclusión, nuestros resultados comparados con los obtenidos por otros autores sugieren que la SG por estadios no es diferente entre los pacientes jóvenes y mayores con CC. Además, la histología no escamosa no es infrecuente entre los jóvenes. Se ha confirmado la relevancia de los factores pronósticos convencionales en pacientes jóvenes con $\mathrm{CC}$, siendo la anemia un factor pronóstico independiente que requiere mayor estudio. A pesar de ser una enfermedad altamente prevenible, la mayoría de nuestros casos fueron diagnosticados en estadios avanzados. Insistimos en la importancia de los programas de cribado integrales para las mujeres 
sexualmente activas y de la vacunación contra el VPH dirigida a niñas y adolescentes antes del inicio de la actividad sexual.

Contribuciones de autoría: RR, MS y LM conceptualizaron y diseñaron el artículo. RR, MS y EFR recolectaron los datos. $\mathrm{RR}, \mathrm{MS}$ y RM realizaron la interpretación de los datos. Todos los autores participaron en la redacción del artículo. NV, MO; MA, LM y HLG realizaron la revisión crítica del artículo. Todos los autores aprobaron la versión final del artículo.

Fuentes de financiamiento: autofinanciado.

Declaración de conflictos de interés: los autores declaran no tener conflictos de interés.

\section{REFERENCIAS BIBLIOGRÁFICAS}

1. Global Burden of Disease Cancer Collaboration, Fitzmaurice C, Dicker D, Pain A, Hamavid H, MoradiLakeh M, et al. The Global Burden of Cancer 2013. JAMA Oncol. 2015;1(4):505-27. doi: 10.1001/ jamaoncol.2015.0735

2. Ferlay J, Soerjomataram I, Ervik M, Dikshit R, Eser S, Mathers C, et al. GLOBOCAN 2012 v1.0, Cancer Incidence and Mortality Worldwide: IARC CancerBase No. 11 [Internet]. Lyon, France: International Agency for Research on Cancer; 2013 [citado el 27 de enero de 2017]. Disponible en: http://globocan.iarc.fr.

3. Ries LAG, Melbert D, Krapcho M, Stinchcomb DG, Howlader N, Horner MJ, et al. SEER Cancer Statistics Review, 1975-2005 [Internet], Bethesda: National Cancer Institute; 2008 [citado el 27 de enero de 2017]. Disponible en: http://seer.cancer.gov/ csr/1975_2005/.

4. Cai HB, Liu XM, Huang Y, Li XN, Lie DM, Zhou Q, et al. Trends in cervical cancer in young women in Hubei, China. Int J Gynecol Cancer. 2010;20(7):1240-3.

5. Kokawa K, Takekida $S$, Kamiura $S$, Kita M, Enomoto T, Kawaguchi R, et al. The incidence, treatment and prognosis of cervical carcinoma in young women: a retrospective analysis of 4,975 cases in Japan. Eur J Gynaecol Oncol. 2010;31(1):37-43.

6. Foley G, Alston R, Geraci M, Brabin L, Kitchener H, Birch J. Increasing rates of cervical cancer in young women in England: an analysis of national data 1982-2006. Br J Cancer. 2011;105(1):177-84. doi: 10.1038/ bjc. 2011.196

7. Baldur-Felskov B, Munk C, Nielsen TS, Dehlendorff C, Kirschner B, Junge J, et al.
Trends in the incidence of cervical cancer and severe precancerous lesions in Denmark, 1997-2012. Cancer Causes Control. 2015;26(8):1105-16. doi: 10.1007/s10552015-0603-7

8. Zhang Y, Shen K, Gao JS, Wu M, Huang HF, Pan LY, et al. [Clinical features and treatment of cervical malignant tumor in young women]. Zhongguo Yi Xue Ke Xue Yuan Xue Bao. 2003;25(4):391-5. Chinese.

9. Rutledge FN, Mitchell MF, Munsell M, Bass S, McGuffee V, Atkinson EN. Youth as a prognostic factor in carcinoma of the cervix: a matched analysis. Gynecol Oncol. 1992;44(2):123-30.

10. Mariani L, Iacovelli A, Vincenzoni C, Diotallevi FF, Atlante M, Lombardi A. Cervical carcinoma in young patients: clinical and pathological variables. Int J Gynaecol Obstet. 1993;41(1):61-6.

11. Lau HY, Juang CM, Chen YJ, Twu NF, Yen MS, Chao KC. Aggressive characteristics of cervical cancer in young women in Taiwan. Int J Gynaecol Obstet. 2009;107(3):220-3. doi: 10.1016/j.ijgo.2009.07.029

12. Yang YC, Shen J, Tate JE, Wang KG, Su $\mathrm{TH}$, Wang KL, et al. Cervical cancer in young women in Taiwan: prognosis is independent of papillomavirus or tumor cell type. Gynecol Oncol. 1997;64(1):59-63.

13. Yang L, Jia X, Li N, Chen C, Liu Y, Wang H. Comprehensive clinicpathological characteristics of cervical cancer in southwestern China and the clinical significance of histological type and lymph node metastases in young patients. PLoS One. 2013;8(10):e75849. doi: 10.1371/ journal.pone.0075849

14. Robertson D, Fedorkow DM, Stuart GC, McGregor SE, Duggan MA,
Nation G. Age is prognostic variable in cervical squamous cell carcinoma. Eur J Gynaecol Oncol. 1993;14(4):283-91.

15. Spanos WJ Jr, King A, Keeney E, Wagner R, Slater JM. Age as a prognostic factor in carcinoma of the cervix. Gynecol Oncol. 1989;35(1):668.

16. Joint World Health Organization/Centers for Disease Control and Prevention (WHO/CDC). Assessing the iron status of populations: including literature reviews: report of a Joint WHO/CDC Technical Consultation on the Assessment of Iron Status at the Population Level [Internet]. 2da ed. Geneva: WHO/CDC; 2004 [citado el 27 enero de 2017]. Disponible en: http://apps.who.int/iris/bitstre am/10665/75368/1/9789241596107_ eng.pdf ?ua $=18 u a=1$.

17. Kidney Disease: Improving Global Outcomes. KDIGO 2012 clinical practice guideline for the evaluation and management of chronic kidney disease. Kidney Int Suppl. 2013;3(1):1-150.

18. Pecorelli S. Revised FIGO staging for carcinoma of the vulva, cervix, and endometrium. Int J Gynaecol Obstet. 2009;105(2):103-4.

19. Webb JA. Ultrasonography in the diagnosis of renal obstruction. BMJ. 1990;301(6758):944-946.

20. Kurman RJ, Carcangiu ML Herrington CS, Young RH, editores. WHO classification of tumours of female reproductive organs, Volume 6. 4ta ed. Lyon, Francia: International Agency for Research on Cancer; 2014.

21. Han CH, Cho HJ, Lee SJ, Bae JH, Bae $\mathrm{SN}$, et al. The increasing frequency of cervical cancer in Korean women under 35. Cancer Res Treat. 2008;40(1):1-5.

22. Elliott PM, Tattersall MH, Coppleson M, Russell P, Wong F, Coates AS, 
et al. Changing character of cervical cancer in young women. BMJ. 1989;298(6669):288-90.

23. Zhao FH, Tiggelaar SM, Hu SY, Xu LN, Hong Y, Niyazi M, et al. A multicenter survey of age of sexual debut and sexual behavior in Chinese women: suggestions for optimal age of human papillomavirus vaccination in China. Cancer Epidemiol. 2012;36(4):384-90. doi: 10.1016/j.canep.2012.01.009

24. Louie KS, de Sanjose S, Diaz M, Castellsagué X, Herrero R, Meijer CJ, et al. Early age at first sexual intercourse and early pregnancy are risk factors for cervical cancer in developing countries. Br J Cancer. 2009; 100(7):1191-7. doi: 10.1038/sj.bjc.6604974

25. Liu L, Sun H. The clinicopathologic characters and prognosis of cervical cancer in 831 young women under 35 years old. Chin Oncol. 2008;18:298-301.

26. Meanwell CA, Kelly KA, Wilson S, Roginski C, Woodman C, Griffiths $\mathrm{R}$, et al. Young age as a prognostic factor in cervical cancer: analysis of population based data from 10,022 cases. $\mathrm{Br}$ Med J (Clin Res Ed). 1988;296(6619):386-91.

27. Davy ML, Dodd TJ, Luke CG, Roder DM. Cervical cancer: effect of glandular cell type on prognosis, treatment, and survival. Obstet Gynecol. 2003;101(1):38-45.

28. Anton-Culver H, Bloss JD, Bringman D, Lee-Feldstein A, DiSaia P, Manetta A. Comparison of adenocarcinoma and squamous cell carcinoma of the uterine cervix: a population-based epidemiologic study. Am J Obstet Gynecol. 1992;166(5):1507-14.

29. Shingleton HM, Bell MC, Fremgen A, Chmiel JS, Russell AH, Jones WB, et al. Is there really a difference in survival of women with squamous cell carcinoma, adenocarcinoma, and adenosquamous cell carcinoma of the cervix? Cancer. 1995;76(10 Suppl):1948-55.

30. Gayan P, Villalobos M, Wendling C, Sierra C, Valencia O, Carcamo M, et al. Survival of cervical cancer type squamous and adenocarcinoma in patients from the National Cancer Institute between 2009-2013, Chile: IGCS-0050 Cervical Cancer. Int J Gynecol Cancer. 2015;25(Suppl 1):17.
31. Gutiérrez C, Alarcón E. Nivel de pobreza asociado al estadio de gravedad del cáncer ginecológico. An Fac Med. 2008;69(4):239-43.

32. Mascarello KC, Zandonade E, Amorim MH. Survival analysis of women with cervical cancer treated at a referral hospital for oncology in Espírito Santo State, Brazil, 2000-2005. Cad Saúde Publica. 2013;29(4):823-31. doi: 10.1590/ S0102-311X2013000800019

33. Instituto Nacional de Estadística e Informática (INEI). Perú: Enfermedades no transmisibles y transmisibles, 2015 [Internet]. Lima: INEI; 2016 [citado el 27 de enero de 2017]. Disponible en: https://www. inei.gob.pe/media/MenuRecursivo/ publicaciones_digitales/Est/Lib1357/ index.html.

34. Bychkovsky BL, Ferreyra ME, StrasserWeippl K, Herold CI, de Lima Lopes G Jr, Dizon DS, et al. Cervical cancer control in Latin America: a call to action. Cancer. 2016;122(4):502-14. doi: $10.1002 /$ cncr.29813

35. Kosary CL. FIGO stage, histology, histologic grade, age and race as prognostic factors in determining survival for cancers of the female gynecological system: an analysis of 1973-87 SEER cases of cancers of the endometrium, cervix, ovary, vulva, and vagina. Semin Surg Oncol. 1994;10(1):31-46.

36. de Rijke JM, van der Putten HW, Lutgens LC, Voogd AC, Kruitwagen RF, van Dijck JA, et al. Age-specific differences in treatment and survival of patients with cervical cancer in the southeast of The Netherlands, 1986-1996. Eur J Cancer. 2002;38(15):2041-7.

37. Grigiene R, Aleknavicius E, Kurtinaitis J. [Prognostic value of anemia for patients with cervical cancer treated with irradiation]. Medicina (Kaunas). 2005;41(11):916-24. Lithuanian.

38. Edge S, Byrd DR, Compton CC, Fritz AG, Greene FL, Trotti A, editores. AJCC Cancer Staging Handbook. $7 \mathrm{ma}$ ed. New York, NY: SpringerVerlag; 2010.

39. Quinn MA, Benedet JL, Odicino F, Maisonneuve P, Beller U, Creasman
WT, et al. Carcinoma of the cervix uteri. FIGO 26th Annual Report on the Results of Treatment in Gynecological Cancer. Int J Gynaecol Obstet. 2006;95(Suppl 1):S43-103.

40. Grigiene R, Valuckas KP, Aleknavicius E, Kurtinaitis J, Letautiene SR. The value of prognostic factors for uterine cervical cancer patients treated with irradiation alone. BMC Cancer. 2007;7:234.

41. Endo D, Todo Y, Okamoto K, Minobe S, Kato $\mathrm{H}$, Nishiyama $\mathrm{N}$. Prognostic factors for patients with cervical cancer treated with concurrent chemoradiotherapy: a retrospective analysis in a Japanese cohort. J Gynecol Oncol.2015;26(1):12-8. doi: 10.3802/ jgo.2015.26.1.12

42. Rose PG, Ali S, Whitney CW, Lanciano R, Stehman FB. Impact of hydronephrosis on outcome of stage IIIB cervical cancer patients with disease limited to the pelvis, treated with radiation and concurrent chemotherapy: a Gynecologic Oncology Group study. Gynecol Oncol. 2010;117(2):270-5. doi: 10.1016/j.ygyno.2010.01.045

43. Harrison L, Blackwell K. Hypoxia and anemia: factors in decreased sensitivity to radiation therapy and chemotherapy? Oncologist. 2004;9(Suppl 5):31-40. doi: 10.1634/ theoncologist.9-90005-31

44. Winter WE 3rd, Maxwell GL, Tian C, Sobel E, Rose GS, Thomas G, et al. Association of hemoglobin level with survival in cervical carcinoma patients treated with concurrent cisplatin and radiotherapy: a Gynecologic Oncology Group Study. Gynecol Oncol. 2004;94(2):495-501.

45. Bush RS, Jenkin RD, Allt WE, Beale FA, Bean H, Dembo AJ, et al. Definitive evidence for hypoxic cells influencing cure in cancer therapy. $\mathrm{Br} \mathrm{J}$ Cancer Suppl. 1978;3:302-6.

\section{Correspondencia: Rossana Ruiz}

Dirección: Av. Angamos Este 2520, Surquillo. Lima, Perú.

Teléfono: (+511) 9888966244

Correo electrónico:Rossana_rm@hotmail.com 